This is a preprint of a submitted author's manuscript that was published in Frontiers in Psychology: Performance Science. This version of the article does not include reviewer or publisher contributions. The final version of the article was published as: Ittner S., Mühlbacher D. and Weisswange T.H. (2020). The Discomfort of Riding Shotgun - Why Many People Don't Like to Be Co-driver. Front. Psychol. 11:584309. DOI: 10.3389/fpsyg.2020.584309

\title{
The discomfort of riding shotgun - Why many people don't like to be co-driver
}

\section{Sandra Ittner ${ }^{*}$, Dominik Mühlbacher ${ }^{1}$, Thomas H. Weisswange (ORCID: https://orcid.org/0000-0003-2119-6965) ${ }^{2}$}

\author{
${ }^{1}$ Wuerzburg Institute of Traffic Sciences GmbH, Veitshöchheim, Germany \\ ${ }^{2}$ Honda Research Institute Europe GmbH, Offenbach, Germany \\ * Correspondence:
}

Sandra Ittner

ittner@wivw.de

Keywords: Information processing, cognition, passenger, comfort, feedback-loop model, situation awareness, risk assessment, autonomous driving

\begin{abstract}
This work investigates which conditions lead to co-driver discomfort aside from classical motion sickness, what characterizes uncomfortable situations and why these conditions lead to discomfort. The automobile is called a "passenger vehicle" as its main purpose is the transportation of people. However, passengers in the car are rarely considered in research about driving discomfort. The few studies in this area focus on driver discomfort, automated vehicles, and on driver assistant systems. An earlier public survey indicated that discomfort is also a relevant problem for co-drivers. An online questionnaire with $\mathrm{N}=119$ participants and a detailed follow up interview study with $\mathrm{N}=24$ participants were conducted.

The results of the online questionnaire show that co-driver discomfort is a widespread problem $(88 \%)$. The results of the interviews indicate that the driving style is the only reason rated as very influential. Frequently mentioned reasons for discomfort are close following or fast driving. Uncomfortable situations were often perceived as safety critical. Participants also felt exposed to these situations. A model for possible cognitive origins of discomfort in co-drivers is proposed based on the study results.

Co-driver discomfort is a common problem, highlighting the relevance of further research on supporting co-drivers. The reported correlations and the extension of theories from the areas of stress and self-regulation can help to explain the origin of this discomfort.

The results provide a foundation for future design of interventions like human machine interfaces aiming at reducing co-driver discomfort.
\end{abstract}

\section{Introduction}

Searching for literature about discomfort in the road vehicle shows that most of it is focused on drivers, even though vehicles are intended to transport multiple passengers. Research about the co- 
driver is very rare, and studies on co-driver discomfort are even fewer. However, a survey by an opinion research institute (Innofact AG, 2013) showed that $77 \%$ of the participants already experienced a situation in which they felt uncomfortable as a co-driver. Ellinghaus and Schlag (2001) conducted a survey, which showed that $19 \%$ of the participants partially or completely agreed that they are frequently afraid of accidents as a co-driver. This indicates that it should be investigated more in detail which factors influence the discomfort of co-drivers in order to derive ideas for interventions, like a human machine interface (HMI), to make their rides more comfortable. Future developments will most likely also lead to higher amounts of passengers in vehicles. Calls for reducing $\mathrm{CO}_{2}$ emissions might increase ride sharing solutions for the general population. Such a solution then consequently leads to higher amounts of passengers in cars. Additionally, with higher automation levels of vehicles, even the driver will become a co-driver in an automated vehicle. It is therefore necessary to examine more closely the conditions under which co-drivers feel uncomfortable and why they do so.

\subsection{Definition of Discomfort}

Similar to emotions feelings like discomfort are a result of a complex evaluation of stimuli. They signal the motivational significance of internal and external stimuli with respect to current goals and needs (Bower \& Cohen, 1982; Lazarus, 1982; Leventhal \& Scherer, 1987). For the co-driver this means that the perceived discomfort signals that something influences their goal of a relaxed ride. Emotions contain reactions on three levels: motoric (muscles, motions), physiological-humoral (CNS, ANS) and subjective-psychological level (feelings). An uncomfortable situation can lead for example to sweating or muscle tension. The Oxford English Dictionary (Stevenson \& Lindberg, 2017) defines "discomfort" as a feeling of "slight pain" or "to make (someone) feel uneasy, anxious or embarrassed". The definition also highlights that discomfort can arise on a physiological level as in the first part of the definition or on a psychological level as in the second part. Discomfort can therefore be measured in different ways. Motion sickness for example can be assigned to physiological discomfort because it includes more physiological symptoms like nausea, sweating, and fatigue (Gianaros et al., 2001). Social discomfort caused by an unusual or unwanted situation is more allocated to the psychological level since symptoms are rather cognitive and emotional, such as embarrassment or stress. But since body and mind are connected, it is important to emphasize that psychological discomfort can still be accompanied by physiological symptoms like muscle tension or abdominal pain. The definition by the Oxford English Dictionary (Stevenson \& Lindberg, 2017) shows also a link to the emotion anxiety. Anxiety is a consequence when a dangerous situation is identified. This leads to the possibility that uncomfortable situations can also be perceived as dangerous or safety critical by co-drivers. Emotions like anxiety or discomfort are also subjective to everyone which means that different stimuli can affect people differently (Drummond et al., 2003). Cosmides and Tooby (2000) described emotions as motivational programs coordinating different behaviors to solve adaptive problems. The fight or flight system will be activated to cope with a situation like the avoidance of enemies or dangers. Such a coping process could be also relevant for co-drivers experiencing discomfort.

\subsection{Explaining the Development of Discomfort}

The transactional stress model by Lazarus and Folkman (1984) is a cognitive model describing the repeating evaluation of situations regarding their threat and the following coping with these situations. The threat of a situation is estimated based upon situational conditions and personal characteristics. Personal characteristics can for example include experiences, personality, or values. Situational conditions are stimuli of the environment with dimension like intensity, duration, and if 
they can be controlled or predicted. If a situation is estimated as threatening or harmful it will be decided if there are enough resources to cope with the situation. The next step would be to decide for a coping strategy. The model states two different ways for this. One strategy is to actively cope with the situation by addressing the threat (problem-focused). The other strategy is more passive, and aims at changing the experienced emotions when it is not possible to change or escape the situation (emotion-focused). This can be for example approached through distraction, avoidance, or denial. The problem-focused strategy will be chosen more likely when a situation seems controllable, and the emotion-focused one when a situation seems less controllable. This means that it strongly depends on the situation which coping strategy is chosen (Folkman et al., 1986).

This cognitive model can help to describe the development of driver and co-driver discomfort and why they sometimes evaluate situations differently. In the transactional stress model, the estimation process of a threat is described for general cases and is not directly related to the regulation of a driving task. Therefore, the transactional model was extended by a feedback-loop model (Carver \& Scheier, 1998; Miller et al., 1960) to understand better which cognitive processes take place during the regulation of a driving task and where sources of discomfort may lie. The feedback-loop model by Carver and Scheier (1998) generally describes the self-regulation process of human behavior. According to the model, someone has a "reference value" or a standard on the basis of which incoming perceptions ("input-function") are compared ("comparator"). If these perceptions differ from the reference value or standard, they will show correcting behavior ("output-function") to adapt their perceptions to the reference value or standard in a looping process. In the next paragraph this model will be combined with the transactional stress model and parallels between both models will be explained. The main purpose of this combination of both models was that the transactional model describes more detailed the coping process. The feedback-loop model describes more detailed the regulation of the driving task by the driver and can show the relevance of information about the cognitive state of the driver during this regulation process for the co-driver.

If both models are mapped to the driver, he/she will evaluate situations based on situational conditions and personal characteristics. In this context situational conditions can be everything having a negative influence on a relaxed and safe ride, like poor road conditions or a high traffic density. Personal Characteristics can be for example their driving experience, their preferred driving style or their personality. The driver influences a situation for example by regulating velocity and distance through iterative adjustments. The driver compares ("comparator") the actual velocity and distance ("input-function" corresponds to situational conditions in the transactional model) with his/her preferred velocity and distance ("reference value" based upon personal characteristics and situational conditions). If there is a discrepancy between actual and preferred velocity and distance, it will be adjusted through a reaction ("output function": corresponds to coping strategy in the transactional model). Since most situations are controllable for the driver the main way to cope with them is the active and problem-focused way, for example by using the brake or gas pedal to increase the distance to other vehicles or changing the lane by using the steering wheel. The results of these reactions can lead to a change in the environment perceived by the driver. The perception-reaction loop is repeated until the preferred velocity and distance are reached. At each point of the feedbackloop, the driver has information about his/her own cognitive state and direct control of the situation, for example through the acceleration/brake pedal. In the less frequent situations where he cannot control the situation the driver is forced to use an emotion-focused coping strategy. This can be for example the case when there is a history of technical problems with the vehicle and the error could not be fixed properly until now. In this situation the driver is not able to predict when another breakdown will occur again. Emotion-focused coping could be then achieved by distracting oneself during driving. 


\section{Related Work}

\subsection{Driver Discomfort}

Searching for situations in which drivers feel comfortable or uncomfortable, it stands out that research often covers vehicle focused factors of situational conditions influencing physiological discomfort rather than psychological factors. A study by Qatu (2012) for example identified noise and vibrations as a main influence on driver comfort, Le et al. (2014) or Hiemstra-van Mastrigt et al. (2017) investigated seating comfort. However, some studies also included psychological discomfort/comfort factors of situations focused on the environment, such as road infrastructure (e.g. complex situations like intersections or roundabouts; Cahour, 2008), other road users (e.g. violent driving style; Dorantes et al., 2016) or the weather (e.g. darkness or skidding; Cahour, 2008). One study identified driving tasks like distance keeping during high traffic density as relevant (de Vos et al., 1997). The study by Constantin et al. (2014) investigated various elements in a vehicle causing discomfort for younger drivers and mapped it to the two dimensions of psychological and physiological discomfort: The seat, the space in the car, and the air condition were mentioned most often for the physical dimension. In the psychological dimension, especially a malfunction of safety relevant elements (e.g., headlights, brakes, or horn) caused discomfort. These situations can be considered as safety critical and uncomfortable since they limit the interaction possibilities with the driving environment.

Personal Characteristics can also influence under which conditions a situation is estimated as safety critical by a driver. The relationship between personality traits, driving style and accident involvement is well investigated (Trimpop \& Kirkcaldy, 1997; Oltedal \& Rundmo, 2006; Sümer, 2003). A study by Iversen and Rundmo (2001) for example showed that drivers scoring high on sensation seeking, driver anger, and normlessness had a more risky driving style. They were also more frequently involved in near-accidents or crashes (with injuries or vehicle damage). This indicates that drivers scoring high on these personality traits and therefore showing a more risky driving style could have a higher threshold for critical situations and experience discomfort in more critical situations than persons scoring lower on these traits.

The studies named in the last two paragraphs indicate that personal characteristics and situational conditions, especially of the areas vehicle and environment, can influence the driver's estimation of the risk in a situation. Most research is focused on the improvement of driver comfort influenced by technical causes. This could be affected by the circumstance that discomfort caused by environmental factors can be easier controlled and influenced by the driver's behavior than most vehicle factors such as the seat or the available space in a vehicle.

\subsection{Co-Driver Discomfort}

Since the co-driver is also part of the ride and interested in a safe and relaxed arrival at a destination, it is possible that the co-driver also evaluates possible threats in a situation. According to the transactional model (Lazarus \& Folkman, 1984) he/she would also base these estimations on the same situational conditions. In contrast to the driver, the role of the co-driver or passenger is mostly passive. The co-driver can monitor the traffic situation but has no direct means of interference. It is also possible for the co-driver to use the center console and to derive information, for example for navigation. The dashboard provides additional driving relevant information like velocity or fuel level, but these are usually not easily accessible to the co-driver. These devices are mainly designed to show information to the driver. The perspective on the environment is slightly different to the driver's visual angle as well, which might result in different percepts (for example distance estimates). The introduced situational conditions for the driver can be similar for the co-driver (e.g. 
road conditions of the area environment or a malfunction of headlights of the area vehicle). All factors, influencing driver discomfort/comfort, could also lead to co-driver discomfort/comfort. However, situations which are uncomfortable for the driver could be even more uncomfortable for the co-driver because of his/her special role in the vehicle caused by differences in available control and information between them.

According to survey results by the opinion research institute Innofact AG the driver can be another situational condition. The survey (Innofact AG, 2013) focused on co-driver discomfort and asked participants which situations caused it. In this survey, $76 \%$ of the participants mentioned that fast driving and close following caused discomfort, followed by false reactions of the driver (60\%) and a distracted driver $(53 \%)$. There is also relevant work in the area of automated driving, where the "driver" has a passive role. These studies also highlight the influence of the driving style of the automated vehicle on discomfort. In a simulator study by Mühl et al. (2019), it was investigated which driving styles of fully automated vehicles or of human drivers are preferred. The participants showed more positive appraisals (e.g. trust ratings) for a human defensive driving style than for a human sporty driving style or either automated driving styles (defensive or sporty). In another simulator study by Griesche et al. (2016) all participants rejected small safety distances and high accelerations. These results were also found in the study by de Vos et al. (1997). The participants rated larger time headways as more comfortable during a ride with a fully automated vehicle. A Wizard of Oz study by Yusof et al. (2016) investigated which autonomous driving styles were preferred in a real driving experiment. The results showed that defensive driving styles were generally preferred. Assertive drivers did not prefer their own driving style when experiencing it during a ride with an automated vehicle. These results can be interpreted that it could be uncomfortable for the co-driver when the driver does not follow a defensive driving style, follows with small distances, or accelerates strongly. It is also possible, that critical values that cause discomfort when being a co-driver might be different from the ones when being a driver. Unfortunately, most studies about comfort or trust during a fully automated ride are either simulator or Wizard of Oz studies with a human driver simulating the automation. This can reduce the feeling of lacking control or can lead to higher trust in automation compared to realistic conditions. There are also no social factors that play a role in research regarding discomfort in automated vehicles. The relationship between driver and co-driver could have an influence on whether the co-driver dares to criticize the drivers driving style.

In contrast to the driver, there is another situational condition for the co-driver: The cognitive state of the driver. This cognitive state is also unknown to co-drivers and can subsequently have an influence on the estimation of the situation's criticality. There can be uncertainty if the driver has his/her attention on the critical situation, if he/she estimates the situation as critical as the co-driver, and the co-driver can be uncertain how the driver will react to this situation. The role of the cognitive state of the driver is comparable to the system state in automated vehicles. The latter often inform the driver about the current state, detected objects, or planned maneuvers through HMIs creating situation awareness in the driver. This can, for example, make it easier for the driver to decide when to take back control of the vehicle (e.g. Naujoks et al., 2017). For the co-driver there are no such HMIs visualizing the driver's cognitive state. A study (Perterer et al., 2015) investigated the benefits of a detailed navigation HMI for co-driver and driver. Besides a map overview, a satellite image, and turn-by-turn instructions, this HMI also displayed information about upcoming hazards. The participants stated that the main advantage of the HMI was that driver and co-driver have the same information about the route and can decide together how to react to certain situations, especially when they are dangerous. Therefore, a more active involvement for example with an HMI could be beneficial for both, the co-driver and the driver, especially when they receive the same information. However, there is no scientific study directly investigating the influence of situational conditions on co-driver discomfort except for the survey by Innofact AG (2013). 
The co-driver's estimation is also influenced by personal characteristics such as their experience as a co-driver or personality. These personal characteristics could influence how easily a situation is estimated as threatening. Ellinghaus and Schlag (2001) showed an influence of personal characteristics, like the experience as a co-driver and the attitude towards being a co-driver on the development of anxiety as a co-driver. The results indicated that more experienced co-drivers and codrivers with a positive attitude towards their role experienced less anxiety. Participants, who rarely are co-drivers and do not like it much, were more afraid of accidents, false reactions of the driver, bad conditions of the car, and felt more bothered having no control as a co-driver. In general, previous studies also showed, that people with higher ratings for neuroticism tend to more likely experience stress or anxiety (Jylhä \& Isometsä, 2006; Gunthert et al., 1999). This could mean that codrivers with higher loads on neuroticism could more likely experience situations as uncomfortable. Beggiato et al. (2015) showed that drivers with higher trust in automation requested less vehicle information about an automated vehicle during a ride. These results indicate that with higher trust it is less necessary to supervise automation and receive control in situations because you trust it to cope with future situations properly. For the co-driver this could mean that the more you trust the driver the more you have trust in him/her to handle situations properly and the less you feel exposed as a co-driver and need less additional information in order to "supervise" the driver. Although there exist studies that did not find an influence of personal characteristics (e.g. Locus of control, Thrill and Adventure Seeking subscale) on the experience of automated driving styles (Bellem et al., 2018), most studies imply that the connection between personal characteristics and the development of codriver discomfort should be investigated.

Looking at the transactional model, the biggest difference between driver and co-driver is the limited possibility to cope with a situation. Since the co-driver has no access to vehicle regulating devices like pedals or the steering wheel there are only two different ways for the co-driver to indirectly cope with the situation actively (problem-focused). One way would be requesting the driver to adapt their driving style to signal an uncomfortable situation. Another way would be to explicitly provide information about the criticality such as a too small distance. This could help the driver to realize the criticality of the situation leading to a feeling of own discomfort and an active coping to reduce it. For both means social factors like the relationship or trust play a role. The co-driver has to rely on the driver to also feel uncomfortable or at least to comprehend that such a situation can be uncomfortable for others. When the driver is reacting to the request, this could have a positive influence on the experienced discomfort and anxiety of the co-driver. When he/she is ignoring it, for example misunderstanding it as criticism of his/her driving style, this could however also have no or an opposite effect. Based on these uncertainties co-drivers could also avoid it to state such a request. When active coping is not possible for the co-driver the last coping strategy would be the passive, emotion-focused way. The co-driver could try to calm down by distracting themselves, pushing an imaginary braking pedal, or by grabbing the door handle. It could be possible that this passive way can also have a positive effect on their discomfort and anxiety (Strentz \& Auerbach, 1988). If these coping strategies are not successful, the co-driver will feel exposed to the situation.

The previous paragraphs have shown the relevance of this topic and that there are hardly any scientific studies investigating conditions causing co-driver discomfort explicitly. The related work about driver discomfort can provide first insights into which conditions could be relevant for codrivers. However, based on the transactional model in combination with a feedback-loop model, major additional differences between driver and co-driver regarding available information and coping might be relevant. Therefore, we have conducted an online questionnaire to provide a first overview on situational conditions of uncomfortable situations causing co-driver discomfort. We focused on the areas: "environment", "vehicle", and added the area "driver" possibly relevant for the co-driver. 
Additionally, we identified frequent co-drivers for additional more detailed interviews about uncomfortable situations as a co-driver through the questionnaire.

In order to propose an extended model describing the development of co-driver discomfort and coping, the influence of personal characteristics, situational conditions further characteristics, as well as which role coping strategies play were considered in the detailed interviews. The results regarding these conditions and characteristics found in the questionnaire and interview are then used as the basis for discussing this extended model. The establishment of co-driver discomfort as a common problem and a proposed model of the cognitive origins enables future work to research means to reduce such negative feelings through technical interventions.

\section{Online Questionnaire}

\subsection{Method}

\subsubsection{Participants}

For the online questionnaire, participants of the Wuerzburg Institute for Traffic Sciences GmbH (WIVW) test panel were recruited via e-mail. The WIVW test panel contains 730 participants from Wuerzburg, Germany and the local area without selection criteria. Participants are between 16 and 94 years and $36 \%$ of the panel are women, $64 \%$ are men. In total, $N=119(60 \%$ women and $40 \%$ men) people participated and completed the online questionnaire. The mean age of the sample was $m=41.28$ years $(s d=15.77$ years). Furthermore, approximately $59 \%$ of the sample reported to be co-drivers 1-3 times a month or less while the other $41 \%$ of the sample were weekly co-drivers (1-2 times a week until daily). Approximately $49 \%$ of the sample were daily drivers the other $51 \%$ drove 3-5 times a week or less.

\subsubsection{Procedure}

The survey was conducted using an online survey tool. Completing the online questionnaire took approximately 10 minutes per participant. The participants had to state if they had experienced any uncomfortable situations as a co-driver. In the introduction it was explained that the questionnaire is focused on psychological discomfort and not on other forms of discomfort caused by nausea (physiological discomfort) or an impolite driver (social discomfort). Afterwards, they had to describe these situations via closed item format questions concerning driver type (e.g. family member, coworker), frequency, and the reasons for discomfort (e.g. fast driving or weather) from the areas driver, vehicle and environment. The answers were presented in randomized order. The reasons for discomfort were requested for each stated driver type in order to allow for a dependent analysis. In the end, the participants could leave their e-mail address and consent that they could be contacted for a following detailed interview regarding co-driver's discomfort.

\subsection{Results}

The results show that $88 \%$ of the participants have experienced at least one uncomfortable situation as a co-driver. The most frequently named driver type in uncomfortable situations is "family members/friends" $(n=97)$, followed with a greater gap by "coworkers/fellow students" $(n=40)$, "taxi drivers" $(n=18)$ and "driver of a lift" $(n=17)$. However, the rate of uncomfortable rides is the highest for "taxi drivers" (33\% of participants named "more than $50 \%$ of rides"), followed by "coworker/fellows" (25\%), "driver of a lift" (18\%) and smallest for "family members/friends" (14 \%). Independent of the driver type, the most frequently experienced reasons are close following, fast driving, and false reactions (Figure 1, left). The pattern is similar when the reasons for discomfort are investigated by driver type. Except for "driver of a lift", the most frequently named reason for 
discomfort is close following, followed by fast driving and false reactions (Figure 1, right). Only for "driver of a lift" fast driving caused most of the co-driver's discomfort.
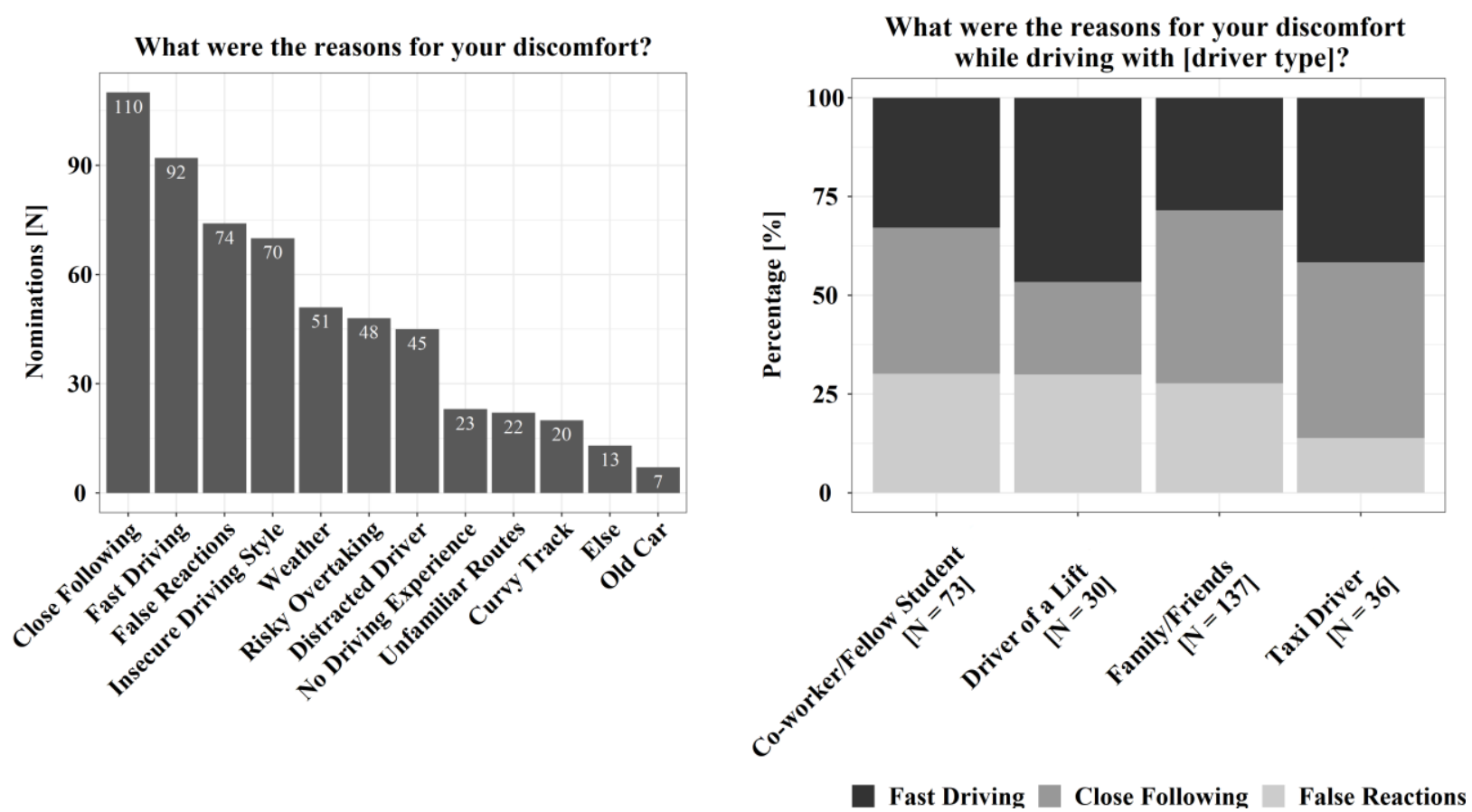

Figure 1. Reasons for co-driver discomfort (left). The three most mentioned reasons for co-driver discomfort by driver type (right).

\subsection{Discussion}

The results of the online questionnaire show that uncomfortable situations as a co-driver are a common problem. The results indicate that co-drivers feel more discomfort with unfamiliar drivers, perhaps due to lower trust in the driver and less experience with the driving style or reactions of the driver. In general, the most frequently named reasons for discomfort were close following and fast driving. These reasons could be allocated to the factor "driver" and are characteristics of the driving style. The remaining reasons could be assigned to the factor "environment" (weather or unfamiliar routes) and the factor "vehicle" (old vehicle). Investigating the factors for discomfort depending on the four different driver types, again the driving style (close following, fast driving and false reactions) was most frequently causing the co-driver's discomfort. The results indicate that the relationship to the driver has a higher influence on how likely discomfort will be experienced than on the reasons leading to discomfort. This may be because a familiar driver can be better assessed. These results match the survey results of the Innofact AG (2013) in which the participants also named fast driving, close following, false reaction and distraction of the driver as main reasons for their discomfort. Furthermore, the results are also similar to the results of the study by Griesche et al. (2016) in which participants mentioned that they dislike small safety distances and high accelerations when driving with an automated vehicle. In the study by de Vos et al. (1997) participants also rated lower distances as less comfortable during driving automated. However, the results differ from the survey results in the co-driver report by Ellinghaus and Schlag (2001). In this report, most participants named malfunctions of the vehicle as main source of anxiety being a co-driver. A possible explanation could be that Ellinghaus and Schlag (2001) focused on anxiety and not discomfort. It could be possible that false reactions of the driver are not perceived as negative as malfunctions of the vehicle. Such malfunctions of the vehicle could lead to more extreme emotions 
like anxiety because they are less frequent, and many people have little experience with technical aspects of a vehicle. The frequently named reasons "close following" and "fast driving" which are characteristics of a more offensive driving style gave an indication that defensive driving styles could reduce the co-driver's discomfort. This can be supported by results for example by Yusof et al. (2016) or Mühl et al. (2019) which showed that defensive driving styles of automated vehicles were perceived as more comfortable.

The online questionnaire contained only closed questions investigating the influence of the three factors "driver", "environment", and "vehicle" on the co-driver's discomfort. The next step was to investigate uncomfortable situations through more detailed interviews. Additionally, the online questionnaire results cannot provide information about the weight of the factors' influence on codriver discomfort and if there is a combination of reasons for discomfort or a single prominent factor. This fact was considered in the interviews through a rating regarding their influence on the co-driver. Furthermore, we considered the influence of personal characteristics on the development of codriver discomfort.

\section{Interview}

\subsection{Method}

\subsubsection{Participants}

In this study $N=24$ participants of the online questionnaire sample (11 male and 13 female participants) with a mean age of $m=46.96$ years ( $s d=12.71$ years) were interviewed. $65 \%$ of the participants were daily drivers, while $35 \%$ were drivers 3-5 times a week or less. $2 / 3$ of the participants were weekly co-drivers, the other $1 / 3$ of the sample were co-drivers 1 to 3 times a month.

\subsubsection{Procedure}

The interview had a duration of approximately 45 minutes per participant. It was semi-standardized and consisted of a protocol with mixed open and closed questions. In the first part, participants answered questions about personal characteristics, and they estimated how often they had experienced uncomfortable situations as a co-driver so far. They were asked about their attitude towards being a driver or a co-driver, if they feel exposed to the traffic and if they prefer overview as a co-driver (6-point Likert scale $1=$ "Does not apply at all" $\ldots 6=$ "Totally agree"). In order to measure the personality trait neuroticism, the participants answered the item "Nervous" ("I easily get nervous and insecure") on a 5-point Likert scale ( $1=$ "Does not apply at all" .. 5 = "Totally agree"). This item is part of the Big-Five-Inventory's (BFI-10; Rammstedt \& John, 2007) "Emotional Stability" scale.

In the second part, participants answered questions about situational conditions and characteristics of concrete uncomfortable situations. For this part, an adapted version of the critical incident technique (Flanagan, 1954) was selected. The participants were asked to recall their most recent uncomfortable situation as a co-driver and give a short summary about this situation. Then the experimenter followed the structured interview. In this interview, the experimenter asked if situational conditions of the "environment", "vehicle", and "driver" had an influence on the uncomfortable situation. If conditions were relevant, the participants rated on a 6-point Likert scale $(0=$ "No influence at all" .. 5 = "Very high influence") how high the influence of these conditions on their discomfort was. They also answered questions about characteristics of the uncomfortable situation. They answered the following questions: how long the discomfort in the situation lasted, if 
they experienced discomfort in the situation on a 5-point Likert scale $(1=$ "Very little" $\ldots 5=$ "Very strong"), if they experienced anxiety, if they felt exposed to the situation, if they trusted the driver and about the criticality of the situations on a 6-point Likert scale $(1=$ "Does not apply at all" $\ldots$ $6=$ "Totally agree").

In the end, the participants named their coping strategies and if those strategies were helpful ("The chosen coping strategy was helpful to reduce my discomfort" rated on 6-point Likert scale from $1=$ "Does not apply at all" .. 6= "Totally agree").

\subsection{Results}

\subsubsection{Personal Characteristics}

The mean fraction of uncomfortable rides as a co-driver was about $20 \%(s d=20 \%)$. With a median $=3.00$ the participants slightly disliked it to be a co-driver (Table 1). However, the results show, that there is almost an even distribution of the ratings over the entire scale showing no clear tendency for this question. The dominant argument to dislike it was because they do not have control as a co-driver, for liking it because it is relaxing. Most of the participants preferred to be driver with a mean rating of $m=5.30$ and the most frequently mentioned reason was that they like it to drive on their own. As co-drivers, they preferred to keep an overview over the surrounding traffic in order to help or warn the driver $(m=4.50)$. They reported feeling slightly exposed to traffic as a co-driver with a mean rating of $m=3.83$, because they cannot intervene in the situation. On the other side there were also participants who fully trusted the driver.

Table 1

Distribution of ratings for personal characteristics (Item) and named reasons for ratings by participants

\begin{tabular}{|c|c|c|c|c|c|c|c|}
\hline \multirow[b]{2}{*}{ Item } & & \multicolumn{3}{|c|}{ Disagree $\leq 3$} & \multicolumn{3}{|l|}{ Agree $\geq 4$} \\
\hline & & Reason & $n$ & $N$ & Reason & $n$ & $N$ \\
\hline \multirow{4}{*}{$\begin{array}{l}\text { "I Like it } \\
\text { to be a co- } \\
\text { driver" }\end{array}$} & \multirow{4}{*}{$\begin{array}{l}m=3.42 \\
s d=1.74\end{array}$} & \multirow{2}{*}{ „I have no control“" } & \multirow{2}{*}{8} & \multirow{4}{*}{14} & „It's relaxing“ & 5 & \multirow{4}{*}{10} \\
\hline & & & & & „I trust the driver“ & 2 & \\
\hline & & $\begin{array}{l}\text { „I do not like the driving } \\
\text { style“" }\end{array}$ & 4 & & „I still like driving“ & 2 & \\
\hline & & Other & 2 & & Other & 1 & \\
\hline \multirow{2}{*}{$\begin{array}{l}\text { "I like it to } \\
\text { be a } \\
\text { driver" }\end{array}$} & \multirow{2}{*}{$\begin{array}{l}m=5.30 \\
s d=0.97\end{array}$} & \multirow[b]{2}{*}{ „I'm not a car fan““ } & \multirow[b]{2}{*}{2} & \multirow[b]{2}{*}{2} & „It's fun/I like driving““ & 15 & \multirow[b]{2}{*}{21} \\
\hline & & & & & „I have control““ & 6 & \\
\hline \multirow{2}{*}{$\begin{array}{l}\text { "I prefer } \\
\text { overview } \\
\text { as a co- } \\
\text { driver" }\end{array}$} & \multirow{2}{*}{$\begin{array}{l}m=4.50 \\
s d=1.53\end{array}$} & \multirow[t]{2}{*}{ „I trust the driver“" } & 3 & \multirow{2}{*}{5} & $\begin{array}{l}\text { „I want overview to help/warn } \\
\text { driver“ }\end{array}$ & 10 & \multirow{2}{*}{19} \\
\hline & & & 2 & & $\begin{array}{l}\text { „I want control “ } \\
\text { Other }\end{array}$ & $\begin{array}{l}2 \\
7\end{array}$ & \\
\hline \multirow{3}{*}{$\begin{array}{l}\text { "I feel } \\
\text { exposed as } \\
\text { a co- } \\
\text { driver" }\end{array}$} & \multirow{3}{*}{$\begin{array}{l}m=3.83 \\
s d=1.58\end{array}$} & „I trust the driver” & 4 & \multirow{3}{*}{9} & „I cannot intervene“" & 12 & \multirow{3}{*}{15} \\
\hline & & $\begin{array}{l}\text { „Depends on driving style } \\
\text { of driver" }\end{array}$ & 3 & & „I trust the driver“" & 1 & \\
\hline & & Other & 2 & & Other & 2 & \\
\hline
\end{tabular}

Note. $m=$ mean, $s d=$ standard deviation.

The results showed no significant relations of co-driver's personal characteristics to the rated discomfort in the situation (Table 2). 
Co-Driver Discomfort

Table 2

Correlations of personal characteristics of the co-driver with their experienced discomfort in the situation

\begin{tabular}{lllc}
\hline Variables $(N=24)$ & \multicolumn{2}{c}{ Discomfort } & Sig. (2-tailed) \\
\hline Sex & Pearson: & $r=-.23$ & n.s. \\
Age & Spearman's rho: $r=-.01$ & n.s. \\
Experience as Co-Driver & Spearman's rho: $r=-.01$ & n.s. \\
Nervous (BFI) & Pearson: & $r=.14$ & n.s. \\
"I Like it to be a co-driver" & Pearson: & $r=-.17$ & n.s. \\
"I prefer overview as a co-driver" & Pearson: & $r=-.31$ & n.s. \\
"I feel exposed as a co-driver" & Pearson: & $r=.02$ & n.s. \\
\hline
\end{tabular}

Note. n.s. $=$ not significant for $\mathrm{p}<.05$.

\subsubsection{Situational Conditions and Characteristics of Uncomfortable Situations}

The most recent situations recalled by the participants were perceived as very uncomfortable, as reflected in a mean discomfort rating of $m=4.00(s d=1.38)$. In these uncomfortable situations, the driver was most often a family member or a friend (approximately $79 \%$ ). The remaining $21 \%$ of cases were with drivers of a lift or of a taxi. The participants also specified how long the feeling of discomfort lasted and $67 \%$ explained that the uncomfortable feeling was limited to a part of the route or a special situation, followed by $21 \%$ feeling uncomfortable during the complete route and $12 \%$ during the complete route and afterwards. Furthermore, in 20 of the 24 named uncomfortable situations, the "driver" was the main factor causing the co-driver's discomfort (Figure 2 left). The participants rated the driving style as highest in influence on their discomfort with a mean rating of $m=3.42(s d=1.93)$, in particular high velocities, close following and an aggressive driving style. Of the remaining uncomfortable situations, three were caused by conditions of the "environment". In these three situations conditions of the "environment road type", especially differing conditions of traffic and infrastructure in the city, on the autobahn or on rural roads were rated as high in their influence. One situation was caused by conditions of the factor "vehicle" (malfunction of the gas pedal). Uncomfortable situations differed significantly from comfortable situations (Figure 2 right). The uncomfortable situations were perceived as clearly safety critical ( $m=5.38, s d=0.71)$ and the participants were afraid of negative consequences like damages $(m=4.21, s d=1.67)$ and injuries ( $m=4.83, s d=1.20)$. Furthermore, the participants felt exposed to the situation $(m=5.08$, $s d=0.83)$. Their trust in the driver was neither clearly high nor very low $(m=3.79, s d=1.53)$. 

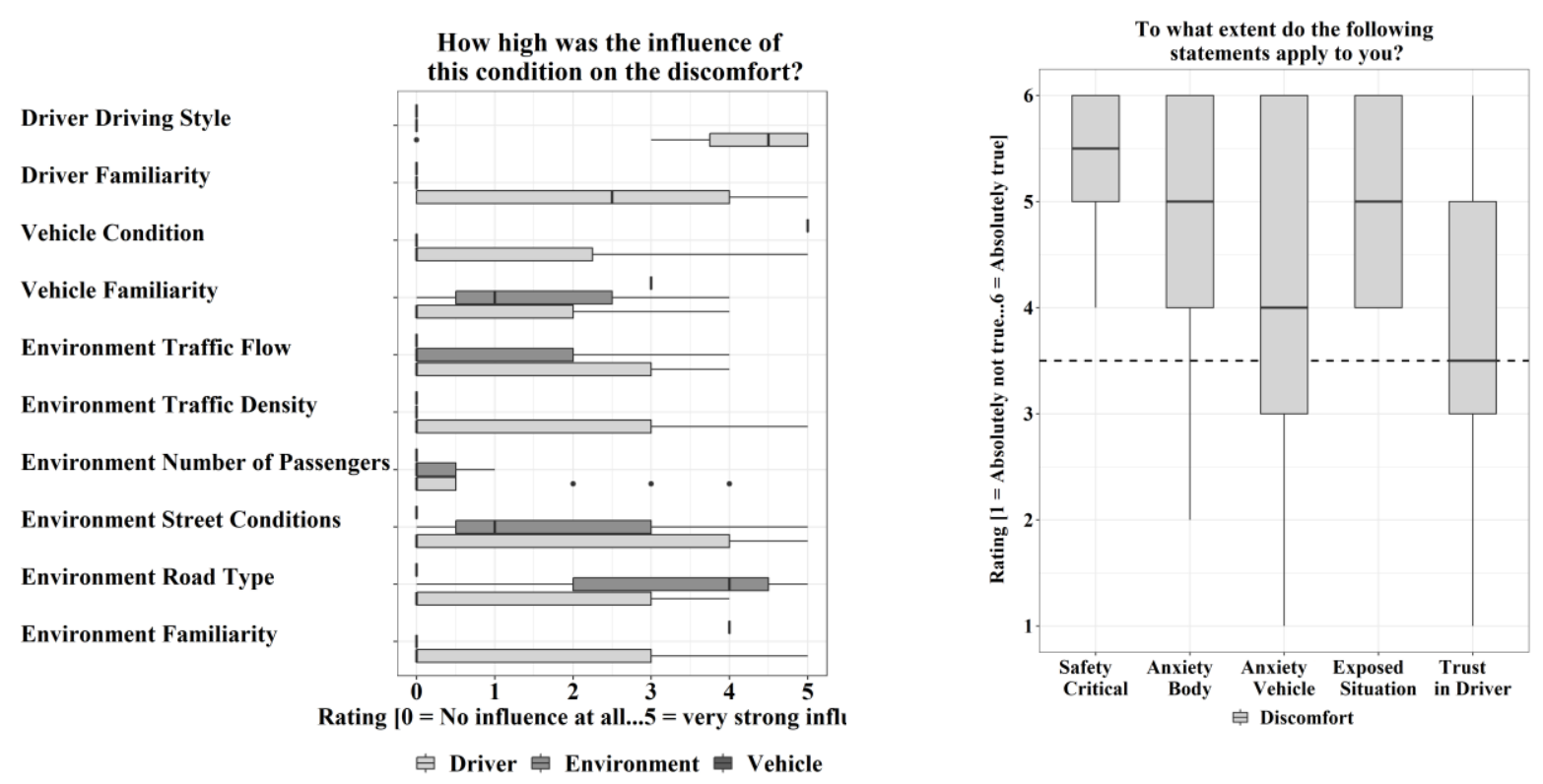

Figure 2. Situational conditions of the three areas and ratings regarding their influence on the discomfort (left). All situational conditions which were rated by more than $75 \%$ of the participants as "not influential" (rating = 0 ) are not presented in the graph. Rated characteristics of their last uncomfortable situations (right).

The correlations (Table 3) indicate that with increasing discomfort, situations were rated as more safety critical $(r=.49)$, participants experienced more anxiety towards injuries $(r=.43)$ or damage to the vehicle $(r=.42)$, and they felt more exposed to the situation as co-driver $(r=.56)$. They also felt more exposed to the situation, when their trust in the driver is reduced $(r=-.43)$.

Table 3

Pearson correlations of characteristics of uncomfortable situations

\begin{tabular}{lccccc}
\hline $\begin{array}{l}\text { Characteristics } \\
(N=24)\end{array}$ & Discomfort & Safety Critical & Anxiety Body & Anxiety Vehicle & $\begin{array}{c}\text { Exposed } \\
\text { Situation }\end{array}$ \\
\hline Safety Critical & $r=.49^{*}$ & - & - & - & - \\
Anxiety Body & $r=.43^{*}$ & $r=.43^{*}$ & - & - & - \\
Anxiety Vehicle & $r=.42^{*}$ & $r=.52^{*}$ & $r=.60^{*}$ & - & - \\
Exposed Situation & $r=.56^{*}$ & $r=.39$ & $r=.36$ & $r=.68^{*}$ & - \\
Trust in Driver & $r=.02$ & $r=-.12$ & $r=.00$ & $r=-.12$ & $r=-.43^{*}$ \\
\hline
\end{tabular}

Note. Significant $(\mathrm{p}<.05)$ correlations are marked with *.

\subsubsection{Coping Strategies}

The participants were asked to name coping mechanisms they used to reduce their uncomfortable feelings (Figure 3). Most of the participants $(N=10)$ named emotion-focused coping behavior like holding the door handle, deep breathing, or distraction, followed by $N=5$ who said something to the driver or criticized him/her (problem-focused coping), $N=5$ who did nothing, and $N=2$ who combined the above strategies. The ratings of the coping mechanism were very mixed showing only a slight tendency of being helpful to reduce their discomfort. $N=2$ participants could not show any coping strategies as the situation was too sudden. 
THE CHOSEN COPING STRATEGY WAS HELPFUL TO REDUCE MY DISCOMFORT

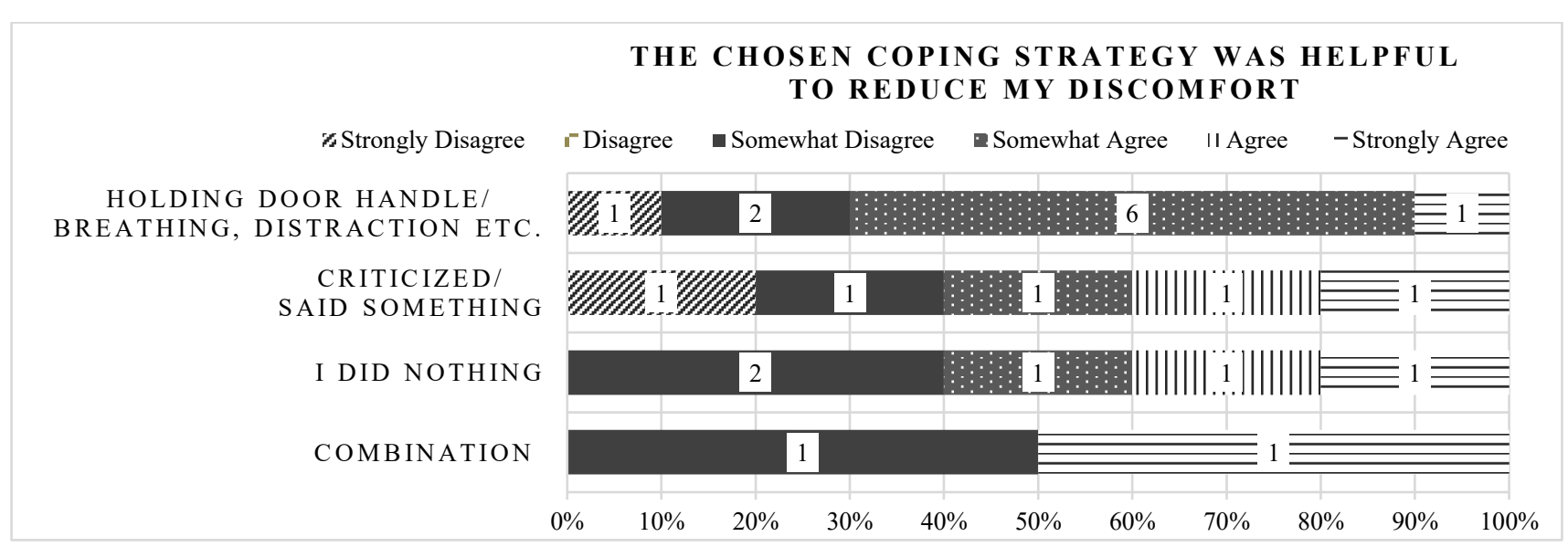

Figure 3. Frequencies of named coping strategy to reduce co-driver discomfort and ratings how helpful they were.

\subsection{Discussion}

The results show that uncomfortable feelings occur most often during specific situations rather than for a whole ride. Uncomfortable situations are perceived as safety critical, almost exclusively caused by the driving style. Furthermore, co-drivers experience anxiety of negative consequences, like an accident, they feel exposed to the situation and have reduced trust in the driver. This indicates that the presence of a situation perceived as safety critical and the missing possibility to actively cope with the situation lead to a feeling of being exposed to the situation and to anxiety of negative outcomes. The feeling of being exposed can increase, if the trust in the driver is low. In the interview, the provided explanations by the participants concerning the statement "I feel exposed as a co-driver" support this conclusion. If they agreed, many participants mentioned that they feel exposed because they cannot intervene, the ones that disagreed explained it by their trust in the driver.

The participants also mentioned how they handled uncomfortable situations and named either strategies focused on changing emotions (e.g. "grasping the door handle", "distraction") or strategies focused on changing the situation (e.g. "asking the driver to keep more distance"). However, the different types of coping strategies were perceived neither clearly helpful nor not helpful in reducing their discomfort. Even the most direct strategy of "asking the driver to keep more distance", seemed only partially effective. This could be caused by drivers who do not follow the requests which could in turn have a negative influence on the trust in the driver.

In contrast to Ellinghaus and Schlag (2001) the influence of personal characteristics like experience as co-driver or the attitude towards being a co-driver on the evaluation of a situation could not be found. One explanation could be that they focused on co-driver anxiety which is a more intense emotion than discomfort. Therefore, it is possible that this focus of the questions produced a greater difference in the groups and answers. They also took their conclusions based on differences in the frequencies of the groups and not based on statistical tests. The results of the present interview however correspond to the findings by Bellem et al. (2018), who similarly found no correlation between relatively general personal characteristics like Locus of Control or Sensation Seeking and the preferred driving style of an automated vehicle. They just found a small relation of the participants own driving style and the preferred automated driving style. However, as participants selected very different uncomfortable situations in the interviews, it would be interesting to investigate the effect of personal characteristics if they would all experience similar situations. 
Co-Driver Discomfort

\section{$5 \quad$ Model of Causes for Co-Driver Discomfort}

Based on the literature and the presented results it is possible to develop an extended model, describing why co-drivers experience discomfort. The left quadrant of Figure 4 shows the driver's regulation and estimation process (comparator) based on personal characteristics (not visualized in Figure 4) and situational conditions, like actual velocity and distance (input-function), with his/her preferred velocity and distance (reference value). However, as Figure $\mathbf{4}$ is modelling the cognitive processes of the co-driver, all parts of the driver's feedback loop that are not accessible to the codriver are crossed out. As mentioned in the introduction it can be assumed that the co-driver also uses most of the same personal characteristics and situational conditions in such a comparison process within his/her own feedback loop (Figure 4 top right). The major difference between the driver's and co-driver's feedback-loop is the fact that the co-driver does not have the possibility to change the actual velocity or distance with a direct action such as braking. There are discrepancies between the amount of information available to driver and co-driver such as limited information about the cognitive state of the driver or a different perspective. This can cause a different estimation of a situation's criticality. This can also lead to the consequence that some situations, which are objectively considered not safety critical, are evaluated as safety critical by the co-driver. This is supported by the assumption that very few drivers would voluntarily keep an uncomfortable driving style or a driving style, which they consider themselves as safety critical, while it might still cause co-driver discomfort. The study by Yusof et al. (2016) showed that assertive drivers did not prefer their own driving style in automated vehicles. Different roles in a vehicle and subsequently different amounts of available information and control can lead to a different estimation of driving styles or situations.

Such safety critical situations can then cause a feeling of discomfort which can be stronger as the situation becomes more critical which is supported by the strong relationship found in the interviews $(r=.49)$. As the definition for discomfort by the Oxford English Dictionary (Stevenson \& Lindberg, 2017 ) indicates, discomfort can be accompanied by anxiety in such an alleged critical situation $(r=.43$ for anxiety regarding injuries and $r=.42$ regarding vehicle damages). After the evaluation of the situation, co-drivers try to cope with the situation either in an active or in a passive and indirect way (Lazarus \& Folkman, 1984). Most of the participants showed any strategy to cope with their discomfort. Only few participants showed no coping strategies or did not have enough time since the situation happened too suddenly. Most of them showed more passive emotion-focused strategies. One explanation could be that these strategies are preferred because they are less offensive trying to avoid conflicts with the driver. All coping strategies showed very mixed helpfulness ratings. Even the problem-focused coping strategy which directly influences the discomfort causing factor showed mixed helpfulness ratings. One reason could be that when the driver is the causing factor and the codriver asks for an adaption of the driving style, he/she must trust the driver that he/she will follow the request. This can limit the co-drivers feeling of having control in the situation. The mixed helpfulness ratings of this coping strategy indicate that there can be drivers ignoring this request.

This situation of being limited to actively cope with the situation or an unsuccessful attempt to cope with it can lead to a feeling of being exposed as a co-driver. This conclusion is not only supported by the relationship found in the interview between their rated trust in the driver and the co-drivers feeling of being exposed in the situation $(r=-.43)$ but also by the answer that they do not generally feel exposed as a co-driver but only in the mentioned uncomfortable situations. The results by Beggiato et al. (2015) support the considerations made in the model that trust in the system/driver can have an influence on how much control and information you want to receive. With higher trust in automation the participants requested fewer information to supervise the system and engaged more in 
secondary tasks, if there was no complex situation. These results indicate that co-drivers could have a higher need for information about the driver's cognitive state and a higher need for more means of active control when the trust in the driver is reduced since they feel more exposed in these uncomfortable situations. The feeling of being exposed can then in turn increase their already existing discomfort and fear. This is also supported by the relationships found in the interviews for these characteristics $(r=.56$ for feeling exposed and discomfort; $r=.68$ feeling exposed and anxiety).

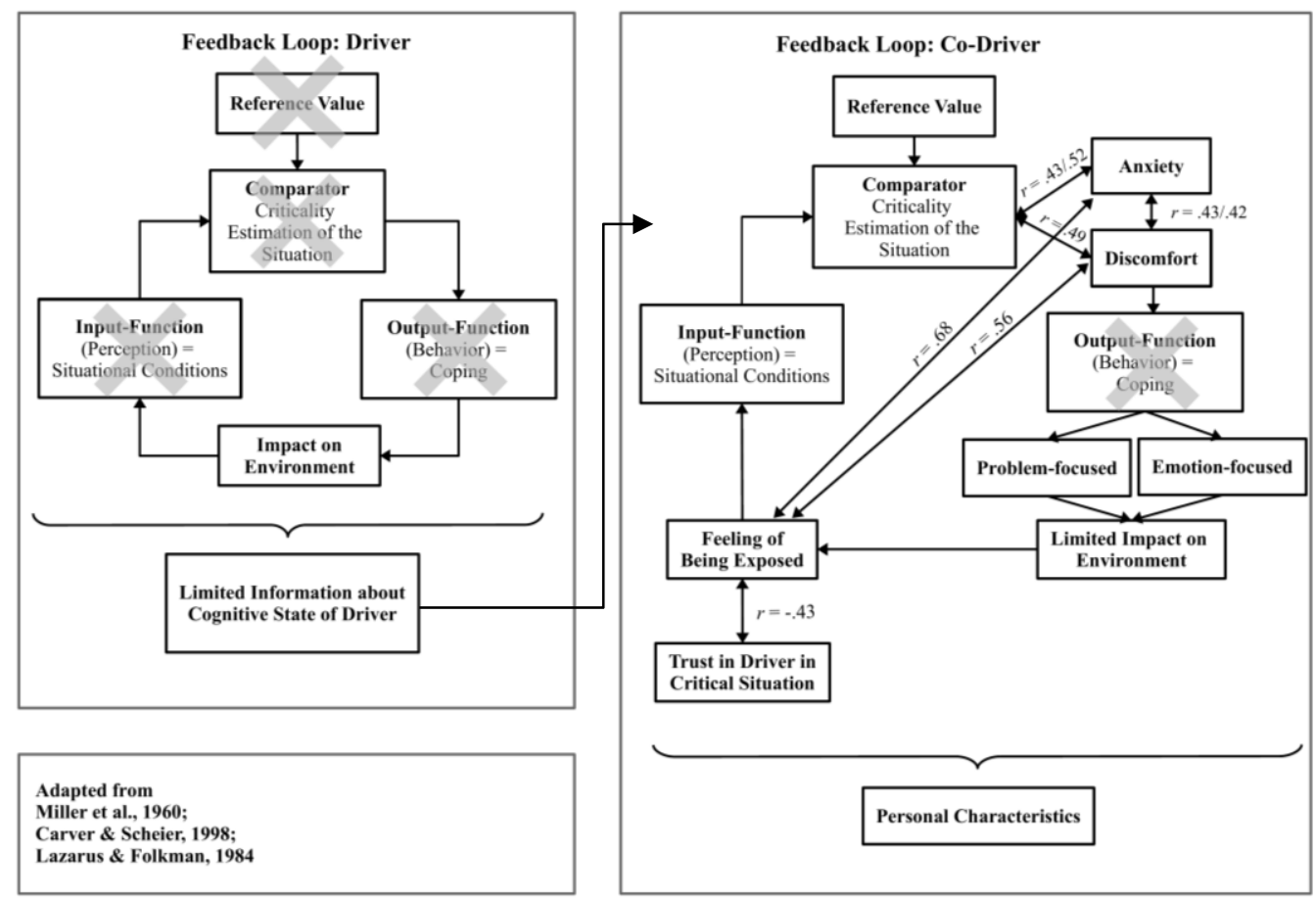

Figure 4. Co-Driver Discomfort Model describing the development of co-driver discomfort considering limited information about the cognitive state of the driver and limited control over the situation. Crossed out are all aspects that are not available or limited to a co-driver. Correlations found in the interview are added to show relations between the components in the model.

\section{General Discussion}

The results of the online questionnaire indicate that co-driver discomfort seems to be a frequently occurring problem. More frequent co-drivers feel uncomfortable in about every 5 th ride. The work in this paper provides evidence that co-drivers should also be considered in the design and evaluation of passenger vehicles. The questionnaire established co-driver discomfort as a common issue and together with the detailed interviews, shed light on possible causes. Uncomfortable situations for the co-driver are mainly and most strongly caused by the driving style of the driver, especially at close following and high velocities. This is in line with the results of the Innofact AG survey (2013) and other studies (Griesche et al., 2016; de Vos et al., 1997; Mühl et al., 2019). In the online questionnaire, the interview and previously in the public survey by Innofact AG (2013) characteristics of the vehicle, like its condition, were rarely mentioned, which is contrary to the results of Ellinghaus and Schlag (2001). Although malfunctions of the car might also cause discomfort, they occur less frequently, which might limit their overall impact on the reports.

The results of the two studies are used to propose a cognitive model that can explain some of the relations and provide opportunities for future detailed investigations. The model is based on two well 
established theories (feedback-loop model and transactional stress model) and extends them to a dyadic co-driver-driver system. In future research, the developed model can be tested by investigating the influence of additional information about the driver's attention or opportunities of active control for the co-driver.

\section{$7 \quad$ Practical Implications}

The results of this work and the developed model could act as a basis for the design of a "co-driver assistant system" showing information or providing control in order to reduce the co-driver's discomfort. Each of the identified aspects of missing information or interaction provides an opportunity for technological intervention. Such interventions can mean that an uncomfortable situation can be turned into a neutral or comfortable one by reducing the perceived criticality. Furthermore, with certain additional information, the co-driver could have a positive effect since he/she could support the driver with the driving task. Such a positive effect of co-driver support was also found in the study by Perterer et al. (2015) and the positive effect of co-driver presence on driving safety was supported by Vollrath et al. (2002). Besides an increase of co-driver comfort such an assistant system could also make it more attractive being a co-driver. The results are also relevant for the development of interfaces in highly automated vehicles when drivers will partially turn to passengers. However, assistance in manual vehicles has additional difficulties when it comes to gathering information about a human driver's internal states. It will therefore be interesting to further research the level of granularity that is sufficient to lower co-driver discomfort in a real vehicle.

Author Contributions: Conceptualization, S.I., D.M., T.W.; methodology, S.I., D.M., T.W.; software, S.I.; validation, S.I., D.M., T.W.; formal analysis, S.I.; investigation, S.I.; resources, S.I., D.M., T.W.; data curation, S.I.; writing-original draft preparation, S.I.; writing-review and editing, D.M., T.W.; visualization, S.I.; supervision, not relevant; project administration, S.I., T.W.; funding acquisition, not relevant. All authors have read and agreed to the published version of the manuscript.

Funding: This project is part of a research program funded and supported by the Honda Research Institute Europe GmbH.

Acknowledgments: We want to thank Alexandra Neukum for administrative support of our experiment.

Conflicts of Interest: This study was conducted as part of a research program of the Honda Research Institute Europe $\mathrm{GmbH}$. Thomas Weisswange is employee of this company. He contributed to the design of the study, review and editing of the manuscript and the decision to publish the results. 


\section{References}

Beggiato, M., Hartwich, F., Schleinitz, K., Krems, J. F., Othersen, I., \& Petermann-Stock, I. (2015). What would drivers like to know during automated driving? Information needs at different levels of automation. Proceedings of the 7th conference on driver assistance. https://doi.org/10.13140/RG.2.1.2462.6007

Bellem, H., Thiel, B., Schrauf, M., \& Krems, J. F. (2018). Comfort in automated driving: An analysis of preferences for different automated driving styles and their dependence on personality traits. Transportation Research Part F: Traffic Psychology and Behaviour, 55, 90-100. https://doi.org/10.1016/j.trf.2018.02.036

Bower, G. H., \& Cohen, P. R. (1982). Emotional influences in memory and thinking: Data and theory. In M. S. Clark \& S.T. Fiske (Eds.), Affect and Cognition (pp. 291-331). Hillsdale, NJ: Erlbaum.

Cahour, B. (2008). Discomfort, affects and coping strategies in driving activity. In J. Abascal, I. Fajardo, \& I. Oakley (Eds.), Proceedings of the 15th European conference on Cognitive ergonomics: the ergonomics of cool interaction, 1-7. https://doi.org/10.1145/1473018.1473046

Carver, C. S., \& Scheier, M. F. (1998). On the self-regulation of behavior. New York, NY, USA: Cambridge University Press.

Constantin, D., Nagi, M., \& Mazilescu, C. A. (2014). Elements of discomfort in vehicles. ProcediaSocial and Behavioral Sciences, 143, 1120-1125. https://doi.org/10.1016/j.sbspro.2014.07.564

Cosmides, L., \& Tooby, J. (2000). Evolutionary psychology and the emotions. Emotions, In M. Lewis \& J.M. Haviland-Jones (Eds.), Handbook of emotions (2nd ed., pp. 91-115). New York: Guilford.

de Vos, A., Theeuwes, J., Hoekstra, W., \& Coëmet, M. (1997). Behavioral aspects of automatic vehicle guidance: Relationship between headway and driver comfort. Transportation Research Record: Journal of the Transportation Research Board, 1573, 17-22. https://doi.org/10.3141/1573-03

Dorantes Argandar, G., Tortosa Gil, F., \& Ferrero Berlanga, J. (2016). Measuring situations that stress Mexicans while driving. Transportation Research Part F: Traffic Psychology and Behaviour, 37, 154-161. https://doi.org/10.1016/j.trf.2015.12.014

Drummond, P. D., Camacho, L., Formentin, N., Heffernan, T. D., Williams, F., \& Zekas, T. E. (2003). The impact of verbal feedback about blushing on social discomfort and facial blood flow during embarrassing tasks. Behaviour Research and Therapy, 41(4), 413-425. https://doi.org/10.1016/S0005-7967(02)00021-9

Ellinghaus, D., \& Schlag, B. (2001). Beifahrer - Eine Untersuchung über die psychologischen und soziologischen Aspekte des Zusammenspiels von Fahrer und Beifahrer. Uniroyal Verkehrsuntersuchung, 26. Köln/Hannover: Continental AG.

Folkman, S., Lazarus, R. S., Gruen, R. J., \& DeLongis, A. (1986). Appraisal, coping, health status, and psychological symptoms. Journal of Personality and Social Psychology, 50(3), 571-579. 
Gianaros, P. J., Muth, E. R., Mordkoff, J. T., Levine, M. E., \& Stern, R. M. (2001). A questionnaire for the assessment of the multiple dimensions of motion sickness. Aviation, Space, and Environmental Medicine, 72(2), 115-119.

Griesche, S., Nicolay, E., Assmann, D., Dotzauer, M., \& Käthner, D. (2016): "Should my car drive as I do? What kind of driving style do drivers prefer for the design of automated driving functions?" 17. Braunschweiger Symposium Automatisierungssysteme, Assistenzsysteme und eingebettete Systeme für Transportmittel, 10(11), 185-204.

Gunthert, K. C., Cohen, L. H., \& Armeli, S. (1999). The role of neuroticism in daily stress and coping. Journal of Personality and Social Psychology, 77(5), 1087-1100. https://doi.org/10.1037/0022-3514.77.5.1087

Hiemstra-van Mastrigt, S., Groenesteijn, L., Vink, P., \& Kuijt-Evers, L. F. (2017). Predicting passenger seat comfort and discomfort on the basis of human, context and seat characteristics: a literature review. Ergonomics, 60(7), 889-911. https://doi.org/10.1080/00140139.2016.1233356

Innofact AG (2013, February 14). Drei von zehn deutschen Beifahrern leiden leise. Repräsentative Umfrage zeigt: Über 40-Jährige vertrauen Fahrer mehr - Frauen bremsen mit. https://www.presseportal.de/pm/13984/2416177.

Iversen, H., \& Rundmo, T. (2001). Attitudes, risk behaviour and accident involvement among Norwegian drivers. Proceedings of the conference Traffic Safety on Three Continents: International conference in Moscow, 18, 12.

Jylhä, P., \& Isometsä, E. (2006). The relationship of neuroticism and extraversion to symptoms of anxiety and depression in the general population. Depression and Anxiety, 23(5), 281-289. https://doi.org/10.1002/da.20167

Lazarus, R. S. (1982). Thoughts on the relations between emotion and cognition. American Psychologist, 37(9), 1019. https://doi.org/10.1037/0003-066X.37.9.1019

Lazarus, R. S., \& Folkman, S. (1984). Stress, appraisal, and coping. New York, NY, USA: Springer.

Le, P., Rose, J., Knapik, G., \& Marras, W. S. (2014). Objective classification of vehicle seat discomfort. Ergonomics, 57(4), 536-544. https://doi: 10.1080/00140139.2014.887787

Leventhal, H., \& Scherer, K. (1987). The relationship of emotion to cognition: A functional approach to a semantic controversy. Cognition and Emotion, 1(1), 3-28.

https://doi.org/10.1080/02699938708408361

Miller, G. A., Galanter, E., \& Pribram, K. H. (1960). Plans and the structure of behavior. New York, NY, USA: Holt. https://doi.org/10.1037/10039-000

Mühl, K., Strauch, C., Grabmaier, C., Reithinger, S., Huckauf, A., \& Baumann, M. (2019). Get Ready for Being Chauffeured: Passenger's Preferences and Trust While Being Driven by Human and Automation. Human Factors, 1-17. https://doi.org/10.1177/0018720819872893 
Naujoks, F., Forster, Y., Wiedemann, K., \& Neukum, A. (2017). Improving usefulness of automated driving by lowering primary task interference through HMI design. Journal of Advanced Transportation, 2017. https://doi.org/10.1155/2017/6105087

Oltedal, S., \& Rundmo, T. (2006). The effects of personality and gender on risky driving behaviour and accident involvement. Safety Science, 44(7), 621-628. https://doi.org/10.1016/j.ssci.2005.12.003

Perterer, N., Meschtscherjakov, A., \& Tscheligi, M. (2015). Co-Navigator: an advanced navigation system for front-seat passengers. Proceedings of the 7th International Conference on Automotive User Interfaces and Interactive Vehicular Applications, 187-194. https://doi.org/10.1145/2799250.2799265

Qatu, M. S. (2012). Recent research on vehicle noise and vibration. International Journal of Vehicle Noise and Vibration, 8(4), 289-301. https://doi.org/10.1504/IJVNV.2012.051536

Rammstedt, B., \& John, O. P. (2007). Measuring personality in one minute or less: A 10-item short version of the Big Five Inventory in English and German. Journal of research in Personality, 41(1), 203-212. https://doi.org/10.1016/j.jrp.2006.02.001

Stevenson, A., \& Lindberg, C. A. (2017). New Oxford American dictionary. Oxford: Oxford University Press.

Strentz, T., \& Auerbach, S. M. (1988). Adjustment to the stress of simulated captivity: Effects of emotion-focused versus problem-focused preparation on hostages differing in locus of control. Journal of Personality and Social Psychology, 55(4), 652-660.

Sümer, N. (2003). Personality and behavioral predictors of traffic accidents: testing a contextual mediated model. Accident Analysis \& Prevention, 35(6), 949-964. https://doi.org/10.1016/S00014575(02)00103-3

Trimpop, R., \& Kirkcaldy, B. (1997). Personality predictors of driving accidents. Personality and Individual Differences, 23(1), 147-152. https://doi.org/10.1016/S0191-8869(97)00017-2

Vollrath, M., Meilinger, T., \& Krüger, H. P. (2002). How the presence of passengers influences the risk of a collision with another vehicle. Accident Analysis \& Prevention, 34(5), 649-654. https://doi.org/10.1016/S0001-4575(01)00064-1.

Yusof, N. M., Karjanto, J., Terken, J., Delbressine, F., Hassan, M. Z., \& Rauterberg, M. (2016). The exploration of autonomous vehicle driving styles: preferred longitudinal, lateral, and vertical accelerations. Proceedings of the 8th International Conference on Automotive User Interfaces and Interactive Vehicular Applications, 245-252. https://doi.org/10.1145/3003715.3005455 